\title{
Modelling Diffusion Effects in Fertility Transition*
}

\section{LUIS ROSERO-BIXBY† AND JOHN B. CASTERLINE}

Mathematical models bind theory to precise formulations, and by doing so accentuate logical inconsistences in theories and facilitate their testing. In this article we explore the implications of simple mathematical models of fertility transition that contain social interaction diffusion effects. We put forward the hypothesis that diffusion effects act as a third type of causal agent of fertility transition, above and beyond the traditionally studied demand and supply factors.

Substantial historical and contemporary evidence points to the existence of diffusion effects on the timing and pace of fertility transition. ${ }^{1}$ Among the pieces of evidence most commonly cited are the fact that fertility declines have occurred under a wide variety of social and economic circumstances, with the pace of decline frequently outpacing the rate of socio-economic change; the irreversibility and rapid pace of decline; the remarkable spatial and temporal patterning of fertility decline in terms of linguistic, ethnic, and cultural boundaries; and, in contemporary developing societies, the relatively small cross-societal and temporal variation in stated reproductive preferences, as compared to the variation in levels of fertility and contraceptive behaviour in the same populations.

While diffusionist explanations are now common in the literature, a lack of conceptual rigour blurs the debate on the role of diffusion dynamics in fertility transition. The possibility that information flows and the diffusion of ideas play a significant role in fertility transition is already mentioned in the earliest fertility studies. Caldwell ${ }^{2}$ complained that 'in the late nineteenth and early twentieth centuries, governments and other institutions almost invariably explained fertility control innovationally, as the spread of pernicious ideas'. Among the earliest fertility transition analysts, Thompson ${ }^{3}$ explained the relatively low fertility of clerical workers in terms of the 'more or less close proximity to their bosses'. An emphasis on the role of the diffusion of ideas characterizes more recent work as well: Cleland and Wilson' posited 'ideational change' as an alternative to explanations centred on material change, and Freedman ${ }^{5}$ described ideas

* This article draws on material in the doctoral dissertation by Luis Rosero-Bixby, Interaction Diffusion and Fertility Transition in Costa Rica (University of Michigan, Ann Arbor, Michigan: 1991). The research was funded in part by Grant number R01-HD-26706 from the U.S. National Institute of Child Health and Human Development.

† Instituto de Investigaciones en Salud (INISA), Universidad de Costa Rica, San José, Costa Rica.

‡ Associate Professor, Department of Sociology, Brown University.

I For a more extensive treatment of the arguments and evidence cited bete, see Casterline et al, 'The Diffusion of Fertility Control', unpublished manuscript. Population Studies and Training Center, Brown University.

2 J. C. Caldwell, Theory of Fertility Decline (New York: Academic Press, 1982), pp, 128-129.

${ }^{3}$ W. S. Thompson, 'Factors in the decline of the birth rate'. In Population Problems (New York: McGrawHill, 1930), p. 127

4 J. Cleland and C. Wilson, 'Demand theories of the fertility transition: an iconoclastic view', Population Studies, 41, 1 (1987), pp. 5-30.

" R. Freedman. "The contribution of social science research to population policy and family planning program effectiveness', Studies in Family Planning, 18, 2 (1987), pp. 57-82. 
and mental frameworks as quasi-independent forces that spread through national and world networks of communication and transportation.

Some authors consider diffusionist explanations of fertility decline as essentially contradictory to motivational theories. Whether couples were able to control their fertility in pre-transition times becomes a key issue. In an influential article, Carlsson ${ }^{6}$ set the debate in terms of whether historical fertility decline was an 'innovation' or just an 'adjustment' to new socio-economic conditions; he interpreted Swedish evidence as supportive of the adjustment hypothesis. In refutation, Knodel ${ }^{7}$ and Watkins ${ }^{8}$ stressed the 'natural' character of pre-transition fertility and the irreversibility of fertility transition as evidence in favour of the innovation hypothesis.

The spread of birth control from the higher social strata to the masses, and the presumed importance of social integration in facilitating or hindering this spread, is another feature of fertility transition labelled as 'diffusion' by many authors. ${ }^{9}$ Without employing the term diffusion, Notestein ${ }^{10}$ stated that "a trend toward birth restriction started in the urban upper classes and gradually moved down the social scale and out to the countryside'.

One outcome of the recent interest in diffusion processes is that the determinants of fertility transition are sometimes dichotomized into those related to socio-economic development and a residue that is tagged 'diffusion'. For example, Retherford and Palmore" stated that 'The timing and speed of fertility transition depend primarily on two factors: economic and social development... and diffusion of birth control' and Bravo ${ }^{12}$ regarded diffusion effects as 'those processes that are different and independent of economic change and economic calculus and that affect decision-making on the number of children'. Alternative conceptual schemes break down this dichotomy by subsuming everything within a diffusion paradigm. Rogers ${ }^{13}$ and his followers view all social phenomena through the prism of innovation diffusion. In the case of fertility behaviour, an individual's acceptance or rejection of family planning is not explained in terms of socio-economic conditions but in terms of the "adoptive potential' of the innovation (i.e. as an essential attribute of birth control) and the individual's 'innovativeness'. Taken to this extreme, diffusion is more a way of thinking than a category of analysis.

In this article, diffusion effects of fertility transition are conceptualized as being distinct from the effects of social and economic variables, but not in opposition to them. Rather, diffusion processes reinforce the effects of social and economic variables. Diffusion processes are so intimately connected to socio-economic forces that ultimately it is impossible to disentangle the two empirically. Nevertheless, certain forms of diffusion effect are conceptually distinct from socio-economic forces, and can be treated

- G. Carlsson, 'The decline of fertility: innovation or adjustment process', Population Studies, 20, 2 (1966), pp. 149-174.

; J. A. Knodel, 'Age patterns of fertility and the fertility transition: evidence from Europe and Asia', Population Studies, 31, 2 (1977), pp. 219.250.

S. C. Watkins, "The fertility transition: Europe and the Third World compared', Sociologieal Forum, 2, 4 (1987), pp. 645-673.

"See, e.g., R. D. Retherford and J. A. Palmore, 'Diffusion processes affecting fertility regulation'. In R. A. Bulatao and R. D. Lee (eds) Determinants of Fertility in Developing Countries, vol. In (New York: Academic Press (1983), pp. 295-339.

${ }^{10}$ F. W. Notestein, 'Economic problems of population change'. In Proceedings of the Eighth International Conference of Agricultural Economists (London: Oxford University Press, 1953), p. 16.

${ }_{11}$ Retherford and Palmore, loc: cit., in fn. 9, p. 295.

12 J. H. Bravo, 'La hipótesis de difusión de la reducción de la fecundidad en Latinoamérica'. Paper presented at Seminar on Fertility Transition in Latin America (International Union for the Scientific Study of Population (In press).

is E. M. Rogers, Communication Strategies for Family Planning (New York: The Free Press, 1973). 
as such in formal models. Indeed, the great value of formal modelling is that it permits cleaner treatment of diffusion effects than is feasible in empirical studies.

In the models developed in this article, we examine diffusion processes that are commonly termed contagion; we prefer endogenous feedback as a descriptor for the behavioural dynamics. The endogenous feedback dynamic occurs when adoption of birth control by some individuals influences the likelihood of adoption by others. Elsewhere we describe behavioural mechanisms that can account for such a dynamic. ${ }^{14}$ In brief, possible mechanisms include; (i) Information flow: knowledge about innovative ideas, technologies, or behaviour can spread from person to person; (ii) Demonstration effect: the experience of some individuals provides concrete evidence for other individuals about the benefits and costs of certain choices; (iii) Changes in normative context : group norms are determined, in part, by the behaviour of group members. In this article we do not explicitly treat what is usually termed 'external source' diffusion processes. These include the spread of information and norms through the mass media, the marketing efforts of professional propagators, and the institutional spread of propagator agencies. In analogy with epidemiological studies, we are concerned here with a process parallel to the spread of diseases by person-to-person contagion, rather than by exposure to fixed hazards such as a contaminated well.

All models are developed in deterministic terms (as differential equations), as opposed to stochastic formulations that are numerically more complex. ${ }^{15}$ The models draw mainly from epidemiological models that describe the sequence of states:

$$
\text { susceptible } \rightarrow \text { latent } \rightarrow \text { infectious } \rightarrow \text { removal }
$$

with neither recovery nor immunity. ${ }^{16}$ The presentation of the models relies on compartmental analysis graphs. ${ }^{17}$ Differential equations that define each model are shown, but no effort is made to find their analytical solution through integration (which in most cases is not attainable anyway). Instead, the presentation consists of numerical solutions obtained with computer simulations. ${ }^{18}$ The simulations were performed using the fourth-order Runge-Kutta method for numerical integration, as implemented in the microcomputer packages Stella ${ }^{19}$ and Phaser. ${ }^{26}$ The simulations are quarter-year steps for numerical integrations.

The purpose of this modelling effort is to clarify concepts, rather than to replicate an observed transition. Keeping models simple, with few parameters, is thus a must. Nevertheless, the formulation of the models (for example, the choice of certain parameter values) is based on an observed fertility transition, that took place in Costa Rica between 1960 and 1976 approximately. During this period, total marital fertility

14 Casterline et al., loc. cit. in fn. 1.

15 J. S. Coleman, Introduction to Mathematical Sociology (London: The Free Press of Glencoe, 1964).

${ }^{16}$ N. T. J. Bailcy, The Mathematical Theory of Infectious Diseases and its Applications, 2nd edn (London: Griffin, 1975). H. W. Hethcote, 'Three basic epidemiological models'. In S. A. Levin, T. G. Hallam and L. J. Gross (eds) Applied Mathematical Ecology, Biomathematics, 18. (Berlin, New York: Springer-Verlag, 1989), pp. $119-144$.

17 J. A. Jacquez, Comparimental Analysis in Biology and Medicine (Ann Arbor, University of Michigan Press, 1985).

${ }_{18}$ A formal treatment, without numerical simulation, of dynamics of the type considered here is contained in $\mathrm{L}$. E. Ediefsen and $S$. I. Ranney. 'An economic model of social norms with an application to fertility'. In Population Association of America (Boston, 1985). R. D. Retherford, "A theory of marital fertility transition", Population Studies, 39, 2 (1985), and, to a lesser extent, N. Crook, 'On social norms and fertility decline', Journal of Development Studies, 14, 4 (1978), pp. 198-210, also develop formal models related to the models in this article.

18 B. Richmond, S. Peterson and P. Vescuso, An Academic User's Guide to STELLA (High Performance Systems, Lyme, New Hampshire, 1989).

${ }^{20}$ H. Kocak, Differential and Difference Equations through Computer Experiments (New York: SpringerVerlag, 1985). 
declined from 7.8 to 3.8 births per woman, and the prevalence of contraceptive use among married women aged $15-44$ increased dramatically from 16 to 59 per cent. ${ }^{21}$ At the same time the proportion who were not using contraception among women who did not want another birth - the 'KAP-gap' - declined in urban areas of Costa Rica from 47 to 32 per cent between 1964 and 1976, and in rural areas from 78 to 38 per cent between 1969 and $1976 .{ }^{22}$

In the first section of this article we present the classical mathematical model for diffusion processes. A dynamic model for what we term the demand-supply paradigm" of fertility transition is proposed thereafter, which subsequently serves as a platform for modelling diffusion effects. In a further section we relax the assumption of homogeneous mix, and allow explicitly for spatial and inter-strata propagation.

\section{THE CLASSICAL DIFFUSION MODEL.}

The classic, deterministic diffusion model describes the adoption of an innovation by a population over time. ${ }^{23}$ Usually the population is assumed closed (i.e. no births or deaths), and individuals are characterized as possessing or not possessing the diffusing attribute. The model is used to describe the progression over time in the cumulative number (or the proportion) of individuals who possess the attribute. This progression usually starts with zero adopters, and ends asymptotically with a pre-established ceiling of potential adopters.

The simplest formulation posits that the number who adopt the innovation per unit of time (i.e. the first derivative $d Y / d t$ ) is a constant fraction of the number of potential adopters who do not yet possess the attribute $(L-Y)$ :

$$
d Y_{t} / d t=a\left(L-Y_{t}\right)
$$

where $Y_{l}=$ cumulative number of adopters at time $t ; L=$ ceiling on the number of potential adopters (susceptibles) and $a=$ constant coefficient of diffusion.

This formulation is known as the 'external-influence' or 'constant-source gains' model. Since the number of potential adopters $(L-Y)$ declines over time, so does the number of new adopters. In turn, the cumulative number $Y$ increases over time according to a Gompertz curve until it reaches the ceiling $L$, which is also the equilibrium level. This model has been used to describe diffusion processes among isolated individuals. ${ }^{24}$ The coefficient $a$ can be interpreted as the inffuence of external agents of change, ${ }^{25}$ such as the effect of mass-media communication, or a newly-opened family planning outlet.

By contrast, the 'internal-influence', or 'interaction-gains', model portrays diffusion as a contagion process exclusively. Spread occurs because those who already possess the attribute (the haves: $Y$ ) transmit it to a constant proportion of the susceptible have-nots $(L-Y)$. The number of new adopters per unit of time is:

$$
d Y_{t} / d t=h Y_{t}\left(L-Y_{t}\right)
$$

where $h=$ constant coefficient of diffusion.

${ }^{21}$ L. Rosero-Bixby and M. W. Oberle, 'Fertility change in Costa Rica 1960-84: analysis of retrospective lifetime reproductive histories', Journal of Biosocial Science, 21, 4 (1989), pp. 419-432, Table 2.

32 V. Gómez, 'Fertility change in Costa Rica: 1964-1986' (University of Wisconsin, 1989, Table 4.7).

${ }^{23}$ Coleman, op. cit., fn. 15, pp. 41-46. V. Mahajan and R. A. Peterson. Models for Innovation Diffusion (Beverly Hills: Sage Publications, 1985). R. R. C. Huckfeldt, W. Kohfeld and T. W. Likens, Dynamic Modeling: An Introduction (Beverly Hills: Sage Publications, 1982), pp. $29-45$.

${ }^{24}$ Coleman, op. cit., fn. 15.

${ }^{25}$ Mahajan and Peterson, loc. cit., fn. 23, p. 15. 
The number of new adopters is small at the beginning of the process, because the sources of contagion $(Y)$ are scarce. Towards the end of the process, the number of new adopters tends to be small again because of the reduced number of susceptibles $(L-Y)$. The cumulative number $Y$ follows the classic logistic curve with asymptotes at zero and $L$, which are also the equilibrium levels of the model. The constant coefficient $h$ represents the effect of horizontal (person-to-person) channels of communication. Isomorphic epidemiological models regard $h$ as a rate of adequate contacts per unit of time. ${ }^{28}$

This pure imitation or contagion model can describe situations in which the only mechanism of diffusion is face-to-face interaction among peers. If external sources of diffusion, such as mass-media or professional propagators, are also acting, the process is better described by a 'mixed-influence model'. The number of new adopters per unit of time in the mixed model is simply the sum of the numbers described by the two previous models:

$$
\begin{gathered}
\left.d Y_{t} / d t=\text { [external source gains }\right]+[\text { interaction gains }], \\
d Y_{t} / d t=\left[a\left(L-Y_{t}\right)\right]+\left[h Y_{t}\left(L-Y_{t}\right)\right] .
\end{gathered}
$$

It can be demonstrated by integration that the cumulative number of adopters $Y$ in this model follows a generalized logistic curve, the shape of which is determined by both coefficients $a$ and $h .^{27}$ There are two equilibrium points: $L$ and $-a / h$ (the second does not have a real-world interpretation). This mixed model seems better suited to study of the spread of birth control in contemporary communities, where both vertical (e.g. family planning programmes, mass media) and horizontal channels of propagation are usually present.

Algebraic manipulations on the discrete forms of these models result in the following recursive formulations:

External-influence model:

Interaction-only model:

$$
Y_{t}=a L+(1-a) Y_{t-1} .
$$

or

$$
\begin{gathered}
Y_{t}=(1+h L) Y_{t-1}-h Y_{t-1}^{2}, \\
Y_{t} / Y_{t-1}=(1+h L)-h Y_{t-1}
\end{gathered}
$$

Mixed model:

$$
Y_{t}=a L+(1-a+h L) Y_{t-1}-h Y_{t-1}^{2} .
$$

These relations can be used to link the models to real data. ${ }^{28} \mathrm{~A}$ time-series in which the number of adopters is a linear function of its one-year lag would suggest an externalinfluence diffusion process. If the plot of this time series against its one-year lag showed a quadratic association, this would suggest pure-interaction or mixed diffusion process, depending on whether the intercept is zero or not, respectively. A pure-interaction model also yields a linear relation between the ratio of two consecutive values of $Y$ and the oneyear lag of $Y$.

It is essential to keep in mind the assumptions of the classic model. The assumption of a closed population holds only for short periods of weeks or months. The process of birth-control adoption, in contrast, usually spans many years, during which the pool of

${ }^{20}$ Hethcote, loc. cit., fn. 16.

27 Mahajan and Peterson, loc. cit, fn. 23, p. 21.

20 The only empirical analyses of the adoption of contraception of which we are aware that have used these formulations are D. W. MacCorquodale and T. W. Pullum, 'A mathematical model for determining the effectiveness of family planning clinics', Studies in Family Plaming, 5, 7 (1974), pp. 232-238, and Mahajan and Peterson, loc. cit., fn. 23, pp. 61-64. 
fecund couples evolves. However, if the renewal process consists of the replacement of mothers by daughters with identical birth-control characteristics (adopters by adopters, susceptibles by susceptibles, and so on), the assumption of static population effectively holds. The classic diffusion model also assumes that coefficients $a$ and $h$ and the ceiling of $L$ potential adopters are constant. These are critical assumptions. Most fertility transition theories are, precisely, about the variation in the number of people susceptible to adopting birth control (the quantity $L$ ) and the determinants of these variations. Moreover, within a context of rapid socio-economic development and dramatic changes in communications, it is highly probable that the diffusion coefficients $a$ and $h$ will also vary. Ignoring these changes can result in serious specification errors.

Interactions occur in this model under conditions of homogeneous or complete mixing. All the probabilities of interacting with, and transmitting the innovation to, all potential adopters are identical for all adopters. In reality these conditions occur only within small communities or selected social networks. As geographers ${ }^{29}$ have observed, individuals have a limited 'mean information field'. The probability of interaction declines with physical and social distance, and individuals vary in their willingness to accept the innovation. These heterogeneities limit severely the applicability of the classic diffusion model.

One might also question the realism of the implicit assumption that the adoption process is binary, i.e. that it occurs in just one stage. Rogers, in contrast, identified four stages in the innovation-decision process: knowledge, persuasion, decision, and confirmation. ${ }^{30}$ The classic model portrays a process in which a population moves from an equilibrium level with no adopters to another equilibrium level in which all potential adopters possess the attribute. The model describes the diffusion process that links these two equilibria. There is no allowance for the process to stall half-way. Furthermore, the timing of the onset of the process and the equilibrium level at the termination of the process - parameters of key interest in the study of fertility transition - are exogenous to the classic model.

\section{A CONVENTIONAL FERTILITY TRANSITION MODEL}

In Figure 1 we present a conventional model of fertility transition in developing countries. For convenience, we term this model the 'demand-supply paradigm', with 'supply' used in a broad sense, as indicated below. The model is intrinsically dynamic: couples move from an initial state of natural fertility into a state of latent demand, and then into a birth control state. $N, L$, and $C$ stand for the number of couples in these three states at time $t$ respectively (for convenience the subscripts $t$ are omitted from the formulae). The sum of the population in these three classes, written by $T$, is the total of fecund couples. The natural-fertility state comprises couples who neither want to, nor do, control their fertility for either spacing or family limitation purposes. ${ }^{31}$ (This definition differs from the standard definition of natural fertility in which deliberate birth-spacing practices are permitted. ${ }^{32}$ ) The latent-demand class consists of couples who want to control their fertility but are not doing so, for whatever reason. The control state consists of those who deliberately regulate their fertility, including couples who are temporarily not practising birth control, because they are seeking a planned birth.

\footnotetext{
20 T. Hägerstrand, Innovation Diffusion as a Spatial Process (English edition) (Chicago: University of Chicago Press, 1967) (Swedish edition, 1953).

${ }^{30}$ Rogers, op. cit, fn. 13, p. 79.

31 H. Leridon. Human Fertility, the Basic Components (Chicago: University of Chicago Press, 1977).

${ }^{32}$ J. Knodel, 'Natural fertility: age patterns, levels, and trends'. In R. A. Bulatao and R. D. Lee (eds), Determinants of Fertility in Developing Countries, vol. I (New York: Academic Press, 1983), pp. 61-70.
} 


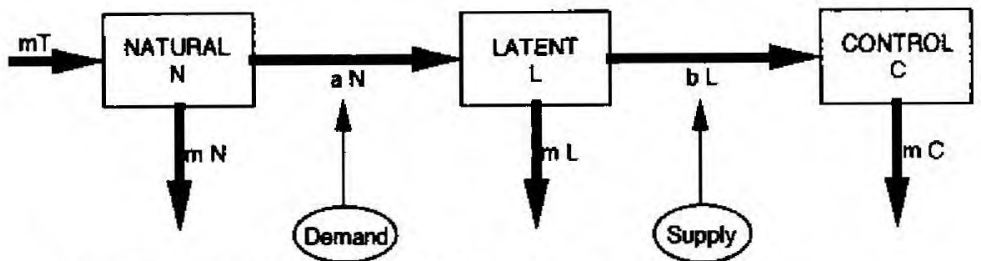

Figure 1. Demand-supply model of birth control adoption.

$m=$ instant renewal rate:

Key relations

$T=$ total population: $N+L+C$;

$d N / d t=m T-m N-a N$,

$a=$ instant rate of demand for birth control;

$b=$ instant rate of access to birth control.

$d L / d t=a N-m L-b L$.

Output summary indicators

,

Proportion of control: $Y=C / T$

KAP gap: $K G=L /(L+C)$,

$d C / d t=b L-m C$.

Total fertility: $F=(1 / m)[0.45(1-Y)+0.04 Y]$

Equilibria for $T=1$

$N^{*}=m /(a+m)$,

$L^{*}=a m /[(a+m)(b+m)]$

$C^{*}=a b /[(a+m)(b+m)]$.

Motivational or demand forces drive the flow between the states of natural fertility and latent demand; costs of birth control (including, but not limited to, the supply of contraceptives) determine the transition between latent demand and birth control. The level of demand determines the relevance of birth control costs: couples have to reach the latent-demand state before cost constraints start to operate. In pre-transition societies the flow from $N$ to $L$ is zero (or almost zero) and the entire population is in a regime of natural fertility. A shift in the motivation or demand forces (e.g. in the socioeconomic or cultural environment) may or may not result in a fertility decline, depending on the cost factors. If there were no cost constraints, no couples would stay in the intermediate state $L$. Non-zero cost constraints keep couples in the latent-demand limbo, a condition known as the 'KAP-gap's3 or the "unmet need for family planning services'. ${ }^{34}$

The model includes vital dynamic: couples enter and exit the system. The arrow on the left and the dark arrows in the diagram's lower part represent these flows. 'Births' or arrivals into this system include marriages and new consensual unions between fecund individuals; 'deaths' or removals are marital disruptions (death of spouse, or divorce) and couples who become sterile. For convenience, the population size $T$ is kept constant by assuming that the instant rates of arrivals and removals are identical and equal to $m$,

$\rightarrow$ the 'renewal rate'. This rate is a function of basic demographic determinants of reproduction (nuptiality, and age and marital-duration structure), which are not of interest in this exercise. The model assumes that $m$ is constant over time and across states.$^{36}$ It follows that the marriage-duration structure is a negative exponential, with the average fecund life equal to $1 / \mathrm{m}$. We employ an annual rate of $m=0.05$, implying 20 years of fecundity, a reasonable figure for societies in which the average age at first marriage for women falls between 20 and 30 .

Couples move from the natural-fertility class $(N)$ to the latent-demand class $(L)$ in quantities proportional to the number $N$, with proportionality constant $a$. This parameter is the 'instant rate of demand for birth control per year'. It expresses the effects of determinants of fertility demand. In turn, the flow from the state $L$ to the birth control class $C$ is proportional to the number $L$, with proportionality constant $b$. This

${ }^{33}$ Freedman, op. cit., fn. 5.

34 C. E. Westoff, 'Is the KAP-gap real?', Population and Development Review, 14, 2 (1988), pp. 225-232.

${ }^{36}$ These assumptions, as well as that of a stationary population, can be easily relaxed, but at the price of introducing more parameters. Perhaps the most important elaboration of the model would be to make $m$ agedependent, but this would introduce considerable complexity without modifying the findings from application of the model to any significant extent. 
parameter, which is the 'instant rate of access to birth control per year', reflects the effects of the costs of practising birth control. (Note that $b$ is an inverse function of birth control costs; keeping in mind their inverse relationship, we will refer interchangeably to 'costs of birth control' and 'access to birth control',)

Fertility transition can be simulated in this model by increasing the parameters $a$ and $b$. Theoretically these parameters can range from 0 to infinity, but a more realistic upper bound is 1.0 . When demand parameter $a=0$, no couples leave the natural-fertility class. In this situation parameter $b$ does not affect fertility levels, since no couples reach the latent-demand state $L$. When demand parameter $a$ is high (say $a=1.0$ ), couples spend little of their reproductive careers in state $N$; they move quickly to $L$, but not necessarily to $C$. If the access parameter $b$ equals 0 , couples who move from $N$ to $L$ will remain indefinitely in state $L$. Note that under these conditions changes in fertility demand will not be reflected in fertility levels. In turn, when the access to birth control is very high (e.g. $b=1.0$ ), couples spend little time in $L$, and, consequently, the extent to which fertility is controlled is determined by motivational forces only.

Figure 1 shows the differential equations that define the model, which are just the net flows represented by thicker arrows in the compartmental graph. The figure also shows the relations at equilibrium for the proportion in each class, which were determined by setting the first derivatives equal to zero and $T=1$. The equilibrium values $N^{*}, L^{*}$, and $C^{*}$ represent intrinsic proportions that correspond to a specific combination of parameters $m, a$, and $b$. The intrinsic proportion in the birth-control state (proportion $C^{*}$ ) is of particular significance. Let us assume that the proportion in the birth-control state required to obtain moderate fertility levels is roughly 0.50 . When birth control is essentially costless ( $b=1.0$ ), $C^{*}=0.50$ if $a=m$ (approximately), that is, an annual rate of demand of about $a=0.05$, given contemporary levels of $m$. Conversely, when birth control is very costly (e.g. $b=0.10$ ), for $C^{*}=0.50$ the demand rate must be about 0.15 . These illustrative figures give some idea of the extent to which parameters $a$ and $b$ must range for the model to represent observed fertility transitions.

The life expectancy in each state provides a further perspective on the implications of this simple model. Couples can expect to spend their reproductive lives (in years) as follows:

$$
\begin{aligned}
\text { All states: } & 1 / m \\
\text { State } N: & 1 /(a+m), \\
\text { State } L: & a /[(a+m)(b+m)], \\
\text { State } C: & a b /[m(a+m)(b+m)] .
\end{aligned}
$$

For example, under the previously described conditions of high costs of birth control $(b$ $=0.10$ ), a demand rate adequate to render $0.50 \mathrm{control}(a=0.15)$, and $m=0.05$, couples would spend five years on average in the natural-fertility class $(N)$, five years as latent demanders $(L)$, and ten years as controllers $(C)$.

Three outcome indicators are defined at the bottom of Figure 1: (1) the cumulative proportion of couples who have adopted birth control, which is denoted $Y$ to maintain the notation of the classic diffusion model; (2) the discrepancy between expressed latent demand and birth-control practice (sometimes termed the 'KAP-gap', hence denoted as $K G)$, which is computed as the proportion of potential adopters $(L+C)$ who are stuck in the latent-demand class $(L)$; and, (3) total fertility $(F)$, which is just a linear transformation of $Y$ for presentation purposes. ${ }^{36}$

\footnotetext{
3. The transformation assumes that the birth rate for couples who do not control their fertility (those in states $N$ and $L$ ) is homogeneous at 0.45 per year (a birth interval of 2.2 years), whereas that for the controllers is homogeneous at 0.04 per year. Total fertility is the product of the weighted average of these two rates and the reproductive life expectancy $1 / m$.
} 
Is the relatively simple mathematical formulation of Figure 1 an adequate representation of the dynamics of contemporary fertility transitions? As an illustration of the adequacy of this formulation, we attempt to reproduce the major features of the Costa Rican fertility transition in Figure 2. Three simulations are generated, that differ in the weight given to the determinants of birth-control demand and birth-control costs: a transition process shaped by both types of factors (Fig. $2 a$ ); a demand-dominated transition (Fig. 2b); and a supply-driven (or costs-driven) fertility decline (Fig. $2 c$ ). For each simulation, $Y, F$, and $K G$ (as well as the parameters $a$ and $b$ ) are plotted against time ${ }^{37}$ Figure $2(d)$ shows corresponding figures for Costa Rica, adapted mainly from the studies of Rosero-Bixby and Oberle ${ }^{38}$ and Gómez. ${ }^{39}$ During the 20 years from the late 1950 s to the late $1970 \mathrm{~s}$, the proportion of couples of reproductive age who controlled their fertility rises from approximately 0.20 to about 0.70 , and total fertility declines from more than seven births to almost three births per woman.

The simulation in which both birth-control demand and costs vary over the course of transition (Fig. 2a) represents perhaps the dominant view in the literature. In this simulation, the initial conditions were set at low levels of demand and accessibility ( $a=$ 0.02 and $b=0.05$ ). In year 5 , the demand parameter soars to $a=0.25$, and in year 15 the access parameter increases to $b=0.20$. The increased demand in year 5 at first generates a surge in the KAP-gap from 0.50 to 0.72 , followed by a slow decline until the increased access to birth control beginning in year 15 plunges it to a new low at equilibrium of 0.20 . The demand-driven simulation (Fig. $2 b$ ) assumes that birth control is relatively accessible throughout the period under consideration, and hence $b$ is fixed at the relatively high level of 0.30 . As in Figure 2(a), the motivation parameter soars from 0.05 to 0.20 in year 5 (reducing total fertility from 7.4 to 4.1 births in ten years). In contrast to this, access improves only slightly in years $15(b=0.35)$ and $25(b=0.40)$, and these changes do not perceptibly affect the trajectory of fertility decline. In response to the sharp increase in demand in year 5 , the KAP-gap surges from 0.14 to 0.43 , but then quickly recedes, and by year 20 returns to its original level. The starting point in the costs-driven simulation (Fig. $2 c)$ is moderate demand $(a=0.10)$ and low access to birth control $(b=0.02)$. Because of the high costs of birth control, a steep increase in the demand parameter has virtually no impact on fertility (nor, interestingly, on the KAP. gap). Conversely, an increase in the access parameter $b$ in year 10 initiates a precipitous decline in both fertility and the KAP-gap; the decline is reinforced with a new improvement in access to 0.20 in year 15 . This simulation terminates at equilibrium levels equivalent to those depicted in Figure 2(a), in which both demand and costs change. Figure $2(c)$, however, illustrates that changes in birth control costs are the proximate cause of fertility transition and that demand factors set the preconditions for a response to changes in costs.

The simulations approximately reproduce the observed time series of Costa Rican total fertility and proportion contracepting (Fig. $2 d$ ). In fact, many other parameter combinations are equally effective. The most decisive conclusion from the three simulations is that a demand-driven process (Fig. $2 b$ ) does not generate the rather high KAP-gap of 0.60 that apparently existed during the early years of the Costa Rican transition. Data on the magnitude of the KAP-gap before 1960 (which do not exist) would provide grounds for choosing between the transition models represented by Figure 2 ( $a$ and $c$ ). According to Figure 2( $a$ ) (demand- and costs-driven), the gap should

\footnotetext{
a7 The renewal rate was assumed constant at $m=0.05$, and the model was initialized at the equilibrium level corresponding to the starting values of the parameters $a, b$ and $m$.

${ }_{38}$ Rosero-Bixby and Oberle, op. cat.. fn. 21.

${ }^{38}$ Gớmez, op. cit., fn. 22.
} 
(a)

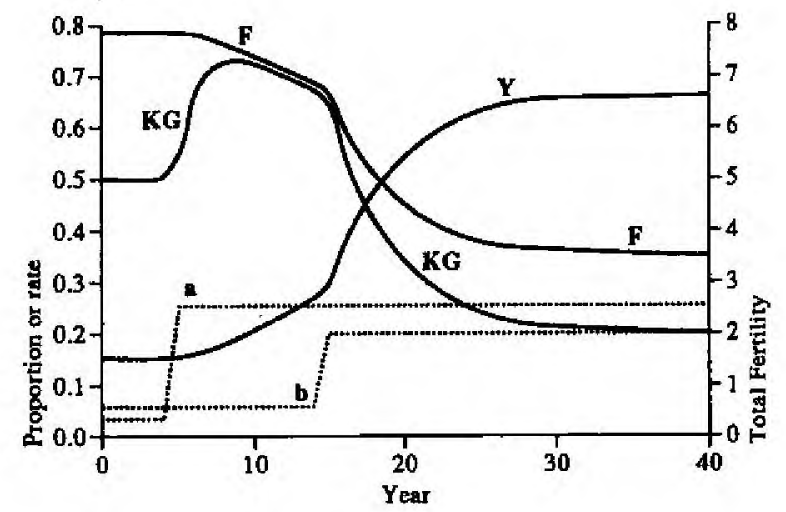

(c)

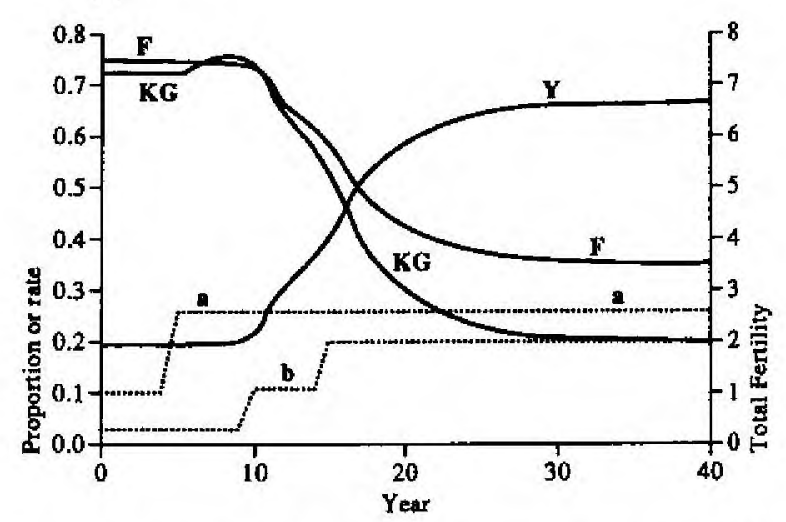

(b)

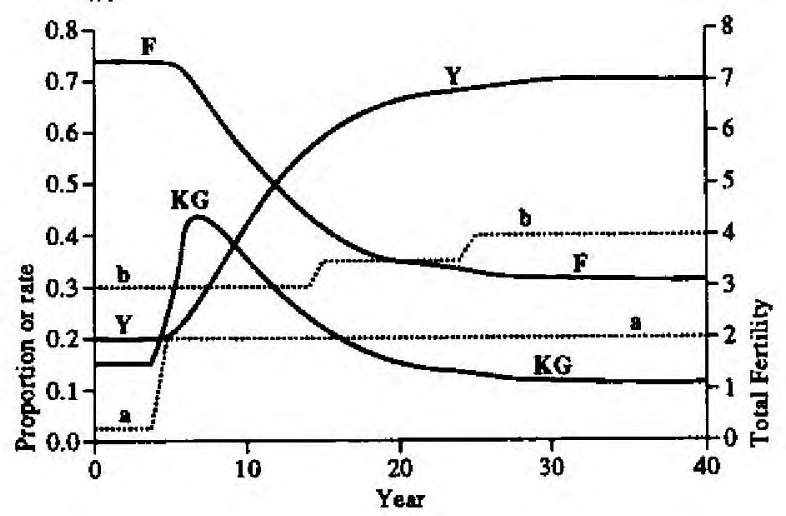

(d)

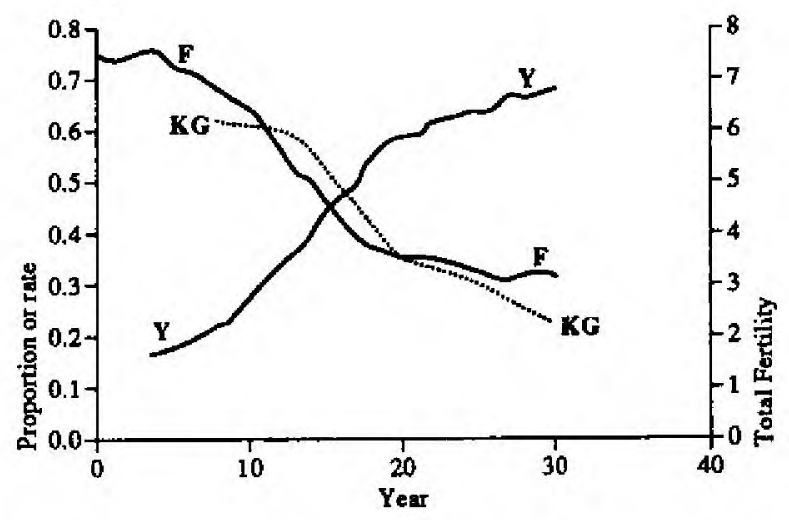

Figure 2. (a) Demand-supply driven simulation. (b) Demand-driven simulation. (c) Supply-driven simulation. (c) Comparison with the observed transition in Costa Rica.

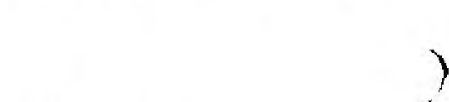


have increased during the very early stages of transition, whereas in Figure 2(c) (costsdriven only) the KAP-gap is high and relatively constant during the early stages.

\section{INCORPORATING INTERACTION DIFFUSION}

The model of Figure 1 can accommodate many of the elements of the traditional innovation diffusion perspective. ${ }^{40}$ For example, a sub-system of the model that explains the access-to-birth-control parameter can easily accommodate the standard diffusionist variables such as adoptive potential, propagator agents, and mass media messages. By the same token, individuals' receptiveness to innovation can be classified as an element of demand. The external-source diffusion coefficient of the classic model ( $a$ in Equation (1)) is, in turn, embedded in the more meaningful parameters of demand and access. This model cannot, however, accommodate internal-influence diffusion, i.e. contagion by social interaction. The model implicitly assumes isolated couples.

Figure 3 adds interaction effects to the demand-supply model. The states of natural fertility $(N)$, latent demand $(L)$, and birth control $(C)$ remain the same, but the flows from $N$ to $L$ and from $L$ to $C$ are now generated in part by contagion from individuals in the birth-control class. The contagion is assumed to refiect the behavioural mechanisms specified above, namely information flow, demonstration effect, and change in normative context. Through one or more of these mechanisms, the rates of transition from $N$ to $L$ and from $L$ to $C$ are determined in part by the proportion of the population in state $C$. That is, the proportion who practise birth control directly influences the likelihood that individuals will move from natural fertility to latent demand and/or from latent demand to birth control. ${ }^{41}$ We assume that social interaction effects are positive; negative effects are theoretically plausible, and are sometimes reported in the literature (e.g. discussion among women about detrimental health effects of certain contraceptives), but the diffusionist arguments presume that positive effects dominate.

In analogy with epidemiological formulations, the model contains the parameter $h$, the instant rate of adequate contacts per year. ${ }^{42}$ An adequate contact of a susceptible is an interaction with another individual which results in adoption of birth control if the other individual is an 'infective'. ${ }^{33}$ The annual number of adequate interactions by the susceptible class is $h L$. Given a probability $C / T$ of interacting with an infective, the number of conversions from $L$ to $C$ due to social interaction is $h L(C / T)$. These conversions add to the costs-determined bL flow of the previous model. The rate of transition from $L$ to $C$ is now $[b+h(C / T)$, i.e. it consists of two components, one of which reflects the effects of social interaction. An analogous reasoning results in the formula for the flow from $N$ to $L$. Note that these relations assume that the population is homogeneously mixing; later we relax this assumption. Finally, Figure 3 also shows the differential equations that define the model ${ }^{44}$ The parameters $a, b$ and $m$ have the same interpretation as in the model of Figure 1.

The model of Figure 3 can be used to simulate the impact of social interaction diffusion on fertility transition. The magnitude of the impact is a direct function, of course, of the selected combination of parameter values. For simulation presented in Figure 4, we choose parameter values that result in a transition that follows a path

${ }^{40}$ As represented, for example, by Rogers, op. cit, fn. 13.

11 The new model assumes that the only 'infective' individuals with the 'family planning virus' are those in state $C$, but contagion properties could easily be added to individuals in $L$ as well.

48 Hethcote, loc. cit., fn. 16.

43 $h$ actually combines two components which are not considered at this level of the model specification: the frequency of contacts, and the probability of transmission by contact.

4 Formulae for the equilibrium points can also be developed but are not shown; the formulae are relatively cumbersome. 


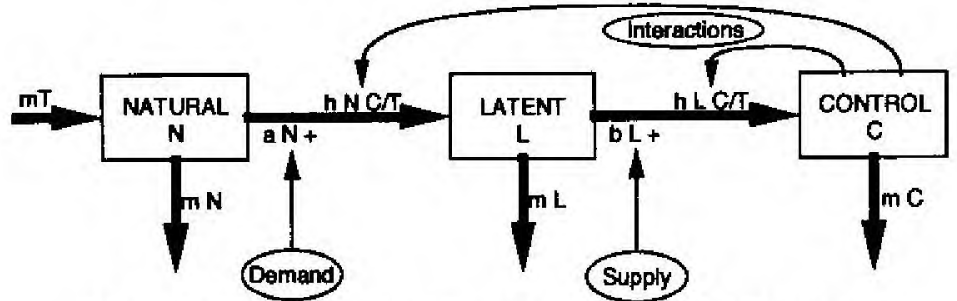

Figure 3. Interaction diffusion model of birth control adoption.

$$
\begin{aligned}
m & =\text { instant renewal rate; } \\
T & =\text { total population: } N+L+C: \\
a & =\text { instant rate of demand for birth control; } \\
b & =\text { instant rate of access to birth control; } \\
h & =\text { instant rate of adequate contacts, including both mean } \\
& \text { number of interactions and a probability of propagation } \\
& \text { per interaction. }
\end{aligned}
$$

Key relations

$d N / d t=m T-m N-a N-h N C / T$,

$\begin{array}{ll}d L / d t=a N+h N C / T-m L-b L-h L C / T, & \text { KAP gap: } K G=L /(L+C) \\ d C / d t=b L+h L C / T-m C . & \text { Total fertility: } F=(1 / m)[0.45\end{array}$

Output summary indicators

Proportion of control: $y=C / T$,

Total fertility: $F=(1 / m)[0.45(1-\eta)+0.04 Y]$.

(a)

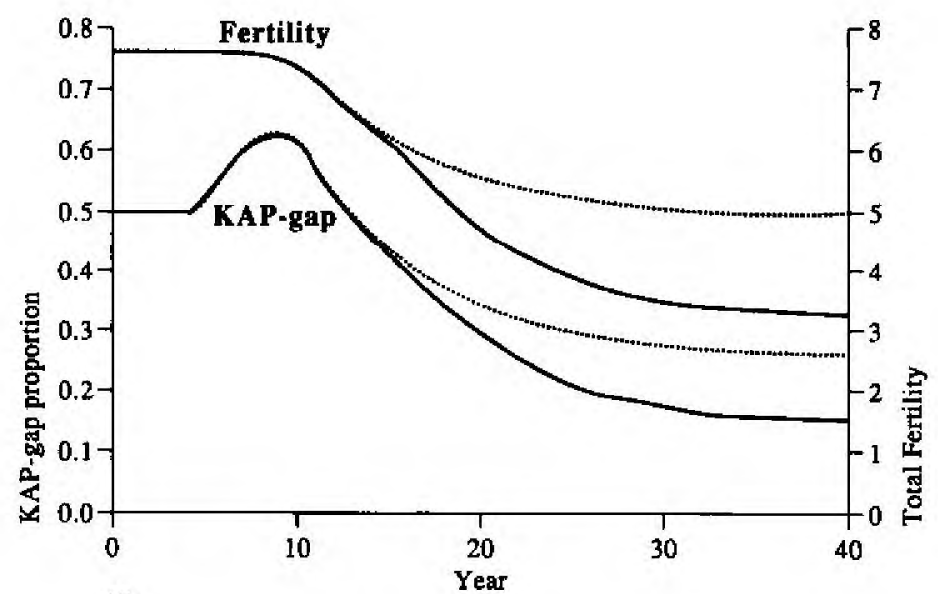

(b)

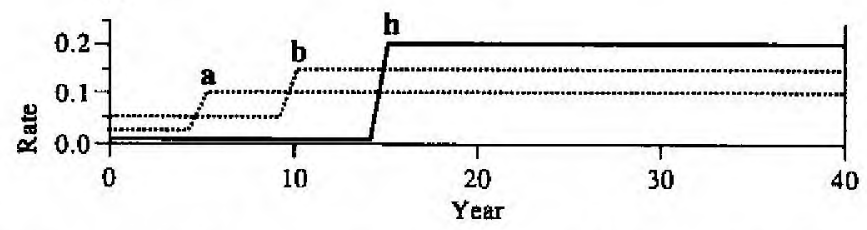

Figure 4. Interaction diffusion model. Simulation of fertility transitions with $(\cdots)$ and without $(\longrightarrow)$ interaction effects.

similar to that observed in Costa Rica, with changes in both demand and cost factors contributing to the transition. Figure $4(b)$ shows the inputs $a, b$ and $h$. Figure $4(a)$ shows two outputs of simulation: total fertility, $F$, and the KAP-gap, $K G$. Dashed lines represent outputs from a model with no interaction effects, i.e. with $h=0$. The difference between dashed and solid lines represents the decline attributable to interaction. 
At this point it may be helpful to step back for a moment and consider the various impacts that interaction diffusion might have on the course of fertility transition. Fertility transitions can be characterized by four variables: (i) pre-transition level of fertility; (ii) timing of onset of decline; (iii) pace of decline; (iv) post-transition level of fertility. Diffusion dynamics are rarely cited with reference to (i), and in this analysis we do not examine in any depth effects on (ii); both types of effects are, however, theoretically plausible. Diffusion dynamics are most commonly invoked with respect to (iii). Less attention has been given to diffusion effects on (iv), but theoretical consistency requires consideration of such effects, because some of the behavioural mechanisms that underlie diffusion effects on the pace of decline would appear to apply with similar force to post-transition levels. This is the case with normative context effects ('social pressure'), which should not be assumed to cease operating during the post-transition phase. ${ }^{45}$ If social interaction can influence post-transition fertility levels, then it becomes difficult, and not terribly meaningful, to distinguish the impact on transition pace from

- the impact on post-transition levels, because transitions to lower fertility levels will be characterized by steeper declines (i.e. rate of decline in fertility per annum). This point will become clearer as the simulations are examined in detail.

Returning to the simulations summarized in Figure 4, note that until year 15 no meaningful independent effects of social interaction are evident, primarily because the contact rate is extremely low $(h=0.01)$. However, once the contact rate rises sharply in year 15, a growing contagion effect emerges. The pace of decline increases (for roughly 15 years, in these simulations), as fertility drops substantially below the no-interaction levels. By the time the system reaches its new equilibrium, interaction diffusion accounts for a reduction in total fertility of almost two births, and in the KAP-gap of more than ten percentage points.

This result illustrates but one of many possible effects of interaction diffusion on fertility transition. The parameters can be combined in many different ways to simulate substantially different effects. How sensitive are the outcomes of these simulations to different parameter combinations? This question is explored in Figure 5 by considering

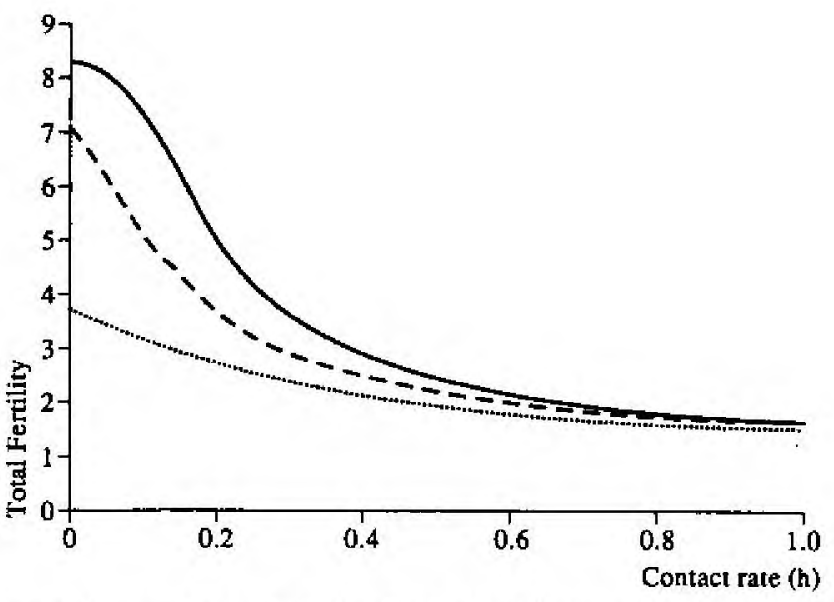

Figure 5. Effects of contact rate on fertility level at equilibrium. Simulations with the interaction diffusion model. - $, a=0.02, b=0.02 ;-\cdots, a=0.02, b=0.20 ; \cdots, a=0.20, b=0.20$.

45 See discussion of the role of social norms in driving fertility to low levels in post-transition societies in Watkins, op. cit., fn. 8. 
how different combinations of the demand and cost parameters condition the repercussions of varying the contact rate $h$. The outcome examined is the total fertility rate at equilibrium. The effect of the interaction feedback is assessed by comparing equilibrium fertility for various values of $h$ against equilibrium fertility when $h=0.0$ (i.e. the ordinate intersection). (Note that each curve in Figure 5 portrays the results of many separate simulations.) When demand is low and costs are high (i.e. the highest curve in Figure 5), a zero contact rate results in total fertility of 8.3 births whereas a contact rate of $h=0.2$ leads to total fertility of 4.9 births; social interaction diffusion thus accounts for a reduction in fertility by $\mathbf{4 1}$ per cent, or almost four births.

Three main findings emerge from the sensitivity analysis in Figure 5.

(1) Interaction diffusion effects are potentially larger under more restrictive demand and costs conditions. For example, in the curve for higher demand and lower costs ( $a=0.2$ and $b=0.2$ ), an increase in the contact rate from 0.00 to 0.20 reduces fertility by 1.04 births ( 27 per cent, as compared to the reduction of nearly four births ( 41 per cent) under conditions of low demand and high costs.

(2) A high contact rate eliminates effects on equilibrium fertility of variations in birth control demand and costs. In Figure 5, the three curves melt together when the contact rate is higher than, say, 0.6. Put another way, contagion effects are capable of breaking down socio-economic, cultural, and even accessibility barriers to birth control. Recall, however, that these simulations assume homogeneous mixing in the population. Thus, we should add the qualification that this finding applies to highly integrated populations (in social and geographical terms).

(3) The marginal effect of the contact rate decreases as the rate increases. For contact rates lower than, say, 0.2 , every increment in the contact rate results in large fertility reductions. In contrast, after the contact rate has reached a value of, say, 0.6 , additional increases have essentially no impact on equilibrium fertility levels.

Note that each of these findings refers to post-transition fertility levels. We repeat our earlier point, however, that effects on post-transition fertility levels will also appear as effects on the pace of transition.

Interaction diffusion is a mechanism through which changes in birth-control costs can create demand. This is a controversial statement in the debate on the causes of fertility transition. The statement follows directly from Figure 3, which shows a feedback path from state $C$ to the transition between states $N$ and $L$; this feedback constitutes in part an impact of birth-control costs on demand, since the size of $C$ is determined in part by parameter $b$. The quantitative impact of this path is illustrated by the solid-line curve in Figure 5 . The curve shows the results of simulations in which demand is set initially at low levels $(a=0.02)$ and access to birth control is set initially at a moderate level $(b=0.20)$. A contact rate of $h=0.1$ results in a post-transition fertility rate of 5.1 births, whereas a contact rate of $h=0.2$ results in 3.6 births. Comparison of these values with a total fertility of 7.1 births for the system in the absence of interaction illustrates how a moderate level of birth control access, in combination with social interaction, can lead to substantial fertility decline.

This result directly reflects the demand-generating dimension of social interaction permitted by this model. Without social interaction - in which case parameter $a$ remains at its initial level of $0.02-$ fertility decline is stymied by inadequate demand. The endogenous feedback process generated by social interaction effectively increases the rate of demand, resulting in substantial fertility decline. To understand that this result is plausible in behavioural terms, recall again the social dynamics identified earlier that might lead to this effect. Movement from state $L$ to state $C$ occurs in this simulation because birth control costs are not prohibitively high. An increase in the proportion of 
the population in state $C$, in turn, increases the rate of movement from $N$ to $L$ (i.e. the demand for birth control) by changing norms about birth control and/or by demonstration of the benefits of controlling fertility and/or because birth control technologies become more widely known. These mechanisms, presume some form of social interaction.

\section{MODELS FOR HETEROGENEOUS POPULATIONS}

The model presented so far assumes homogeneous mixing in the population, i.e. that the probability of interacting with any other member of the population is equal for all individuals. This assumption clearly runs counter to the fact that social interaction is seriously constrained by spatial and social distances. Heterogeneity can be introduced into the model relatively simply by stratifying the population.

In Figure 6 we present a model for the simplest case of heterogeneous mixing, consisting of just two strata. Although this constitutes an unrealistically simple social structure, the model appears substantially more complex. The complexity is more apparent than real, however. All relations within each stratum are replicas of those in the previous, homogeneous model. The only new elements in the model are the paths which represent interactions between strata, the strength of which is captured by parameter $g$, the rate of adequate contact between strata. This rate should logically be smaller than the within-stratum contact rate $h$. Flows from $N$ to $L$ and from $L$ to $C$ thus now contain a third term, which accounts for the interactions between strata. For example, the flow from natural fertility to latent demand in the second stratum includes a third term, which equals the number of inter-strata contacts $(g N)$ times the probability $\left(C^{\prime} / T\right)$ of contacting an 'infective' (the prime denotes the first stratum). The differential equations that define the model are shown at the bottom of Figure 6.

Figure 7 simulates the functioning of the two-strata model. Low levels of birth-control demand and costs $\left(a=a^{\prime}=0.025\right.$ and $\left.b=b^{\prime}=0.05\right)$ obtain at the beginning of the simulation. These rates remain constant in the second stratum, but in the first stratum they increase to $a=0.10$ and $b=0.20$ in years 5 and 10 , respectively. The intra-stratum contact rate is constant at $h=0.15$. To illustrate the effect of inter-stratum contagion, the contact rate $g$ is set to 0.00 at the beginning and then is increased to 0.05 in year 30 . Note that we assume that inter-strata contact rates are far smaller than intra-stratum contact rates. Figure $7(a)$ shows the stratum-specific fertility rates generated by this simulation. It also shows, with dashed lines, the outcome under the condition of no social interaction throughout the simulation period $(h=0$ and $g=0$ ). The difference between the dashed and solid lines constitutes the combined effects of intra- and interstrata interaction.

Fertility in stratum 2 declines from 7.6 to 4.3 births. The whole of this decline can be attributed to contagion effects, which increase the transition rates from $N$ to $L$ and from $L$ to $C$ beyond the rates $a$ and $b$, which remain constant at low levels. A change in the slope of the solid curve for stratum 2 is evident in year 30 , when the inter-strata contagion effect begins to act. This effect accelerates the fertility decline and reduces fertility to 4.3 births in year 60 . A simulation in which parameter $g$ remains at 0.00 (not shown in the Figure) would yield a figure with one fewer birth. Within-stratum contagion accounts for an additional reduction in fertility by 2.2 births at year 60 , so that the total decline in fertility attributable to contagion is 3.2 births. These results illustrate how plausible social interaction processes can have substantial effects on fertility rates and the trajectory of fertility transition.

The spread of fertility control from an elite to the masses and from a centre to the 


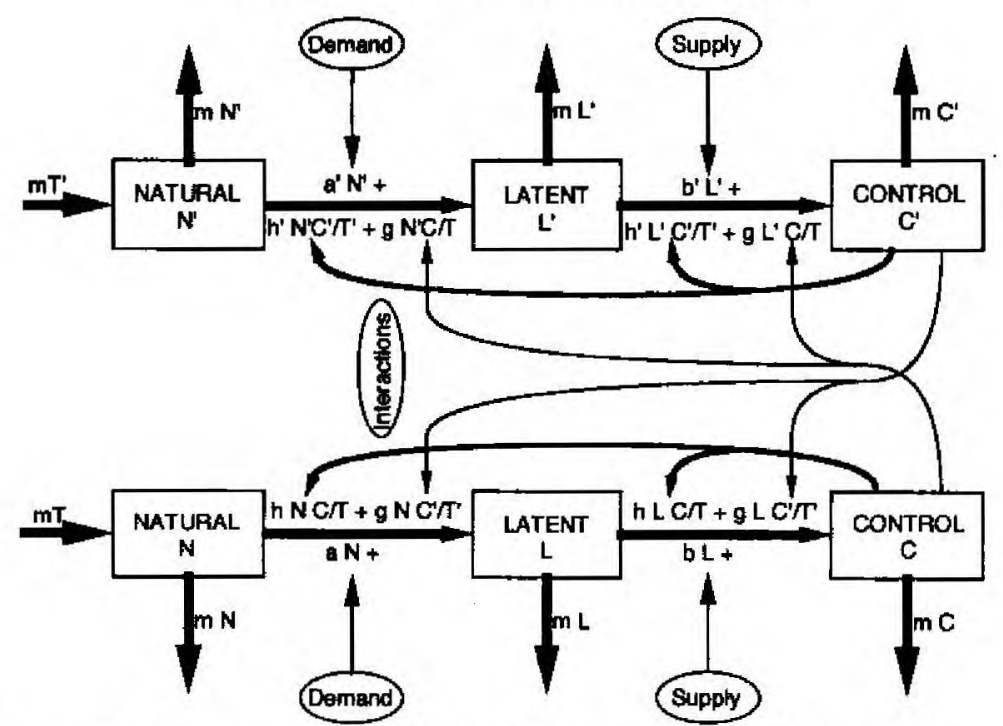

Figure 6. Heterogeneous mix, interaction diffusion model of birth control adoption.

$m=$ instant renewal rate;

$T=$ total population: $N+L+C$;

$b=$ instant rate of access to birth control;

$a=$ instant rate of demand for birth control;

$$
\begin{aligned}
& h=\text { instant rate of adequate contacts within stratum; } \\
& g=\text { instant rate of adequate contacts between strata. }
\end{aligned}
$$

Key relations for the first stratum

$$
\begin{aligned}
& d N^{\prime} / d t=m T-m N^{\prime}-a^{\prime} N^{\prime}-h^{\prime} N^{\prime} C^{\prime} / T-g N^{\prime} C / T, \\
& d L^{\prime} / d t=a^{\prime} N^{\prime}+h^{\prime} N^{\prime} C^{\prime} / T+g N^{\prime} C / T-m L^{\prime}-b^{\prime} L^{\prime}-h^{\prime} L^{\prime} C^{\prime} / T-g L^{\prime} C / T, \\
& d C^{\prime} / d t=b^{\prime} L^{\prime}+h^{\prime} L^{\prime} C^{\prime} / T+g L^{\prime} C / T-m C^{\prime} .
\end{aligned}
$$

Key relations for the second stratum

$$
\begin{aligned}
& d N / d t=m T-m N-a N-h N C / T-g N C^{\prime} / T, \\
& d L / d t=a N+h N C / T+g N C^{\prime} / T-m L-b L-h L C / T-g L C^{\prime} / T^{\prime}, \\
& d C / d t=b L+h L C / T+g L C^{\prime} / T-m C .
\end{aligned}
$$

periphery is often cited as evidence of diffusion effects. Figure 8 shows simulations aimed at studying this trickle-down effect. The underlying model contains three strata. Stratum 1 serves as the source of contagion. There are interactions between strata 1 and 2, and between 2 and 3, but not directly between the first stratum and the third. Demand and costs in stratum 1 are initially more conducive to fertility control (values of parameters $a$ and $b$ of 0.04 , as against 0.01 in the other strata). The initial equilibrium level (allowing for intra-stratum social interaction effects) is a total fertility of $5.8,6.8$, and 7.7 births, in stratum 1, 2 and 3, respectively. (In Latin America and some other societies, pretransition fertility was lower among the urban middle classes than in the remainder of the population.) In these simulations, the demand and access parameters sharply increase to 0.20 in stratum 1 as of year 5 , while remaining constant at 0.01 in the other two strata. Two simulations are carried out: one with constant contact rates (Fig. 8a), and a second with an increase in these rates in the tenth year (Fig. $8 b$ ).

The change in demand and access, magnified by contagion effects, generates a rapid fertility transition in stratum 1 (curves F1 in Fig. 8). The resulting new equilibrium is 3.2 or 2.4 births, depending on the specified contact rates. Because of inter-strata social interaction, this fertility decline in stratum 1 provokes fertility decline in stratum 2 and, indirectly, in stratum 3 as well. The contagion process takes dramatically different forms 
(a)

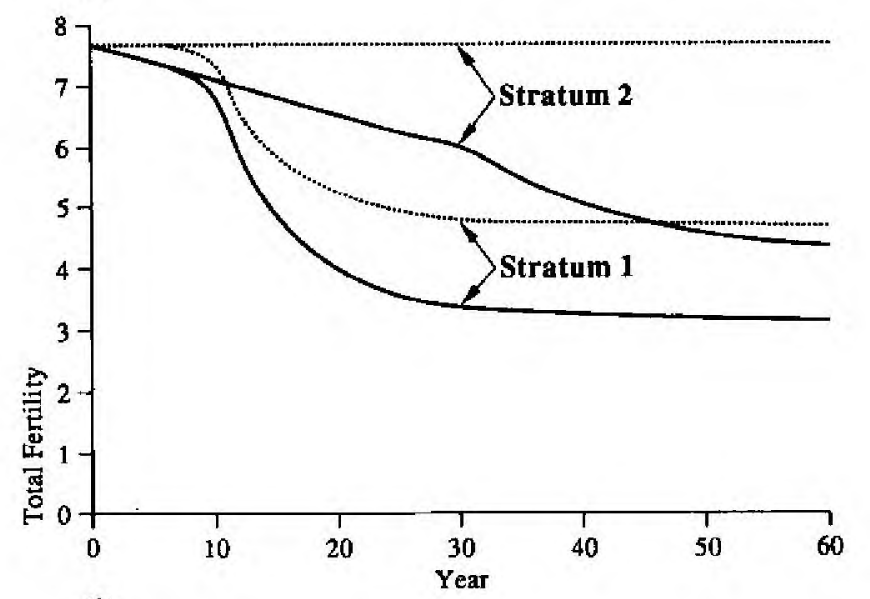

(b)

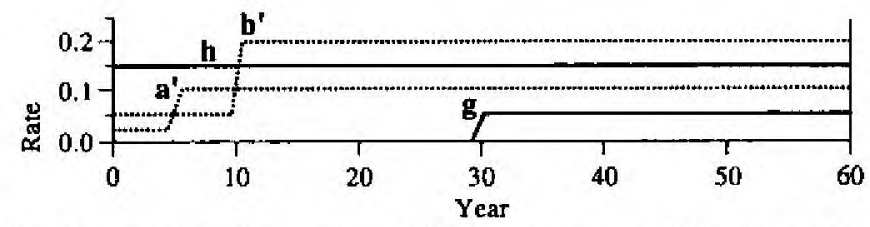

Figure 7. Fertility transition simulation with the heterogeneous mix, diffusion model. - , No interaction; $\cdots$, with interaction. Note that $a=a^{\prime}$ until year 4 , and stays constant the whole period; $b=b^{\prime}$ until year 9 , and stays constant the whole period.

in the two simulations. Under constant contact rates (Fig. $8 a$ ), strata 2 and 3 are only slightly influenced by the fertility decline in stratum 1 , because contact rates remain relatively low and constant. In contrast, strata 2 and 3 respond with sharp fertility declines when contact rates increase (Fig. $8 b$ ); the equilibrium fertility falls to 3.1 births in stratum 2 and to 3.9 births in stratum 3.

These results disclose a critical feature of diffusion dynamics that has not been stressed in the literature on fertility transition: to have a substantial impact on the pace of transition and/or post-transition fertility levels, social interaction probably must increase markedly during the period of transition. This outcome obtains for the following reasons. On the one hand, contagion effects cannot be strong before the transition, because this is not compatible with the co-existence of a high overall level of fertility and a nucleus of couples controlling fertility: high contact rates would have led to the diffusion of birth control to the rest of the population a long time ago. ${ }^{46} \mathrm{On}$ the other hand, if the low pre-transition contact rates remain unchanged, diffusion effects are modest (Fig. $8 a$ ).

The fertility differentials shown in Figure $8 b$ illustrate the profile that may be expected when a trickle-down process takes place: differentials widen during a first stage of the transition and converge during a second stage. Furthermore, the simulations illustrate the net effect of inter-strata distances on the pattern of differentials over time. The basic parameters, of strata 2 and 3 are identical but stratum 2 has direct contact with stratum 1 (whereas stratum 3 has contact with stratum 1 only indirectly, through stratum 2). Greater proximity to the source of contagion results in lower fertility levels in stratum

${ }^{46}$ For this reason, the simulation shown in Figure $8 a$ uses relatively low constant contact rates, because otherwise the initial equilibrium fertility rate would have been unrealistically low. 
(a)

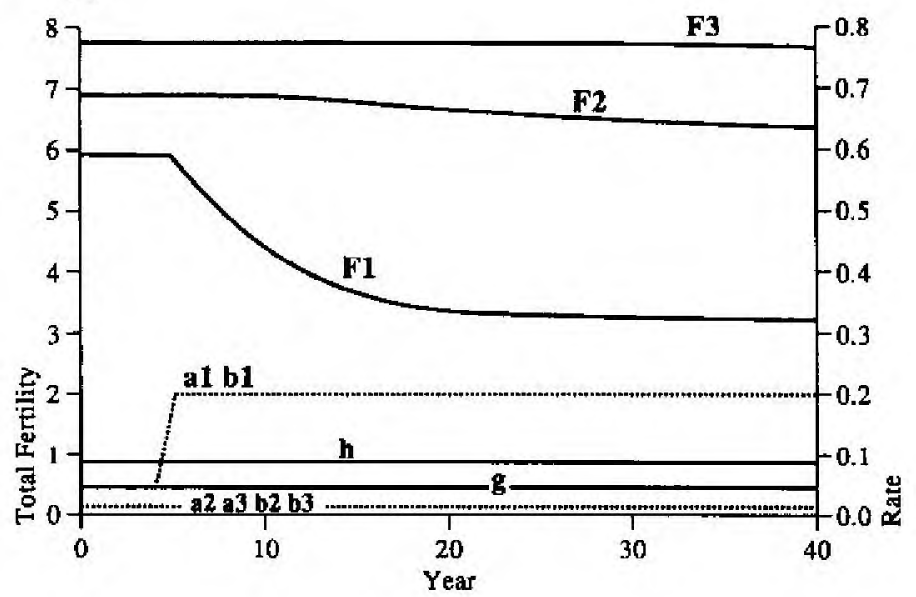

(b)

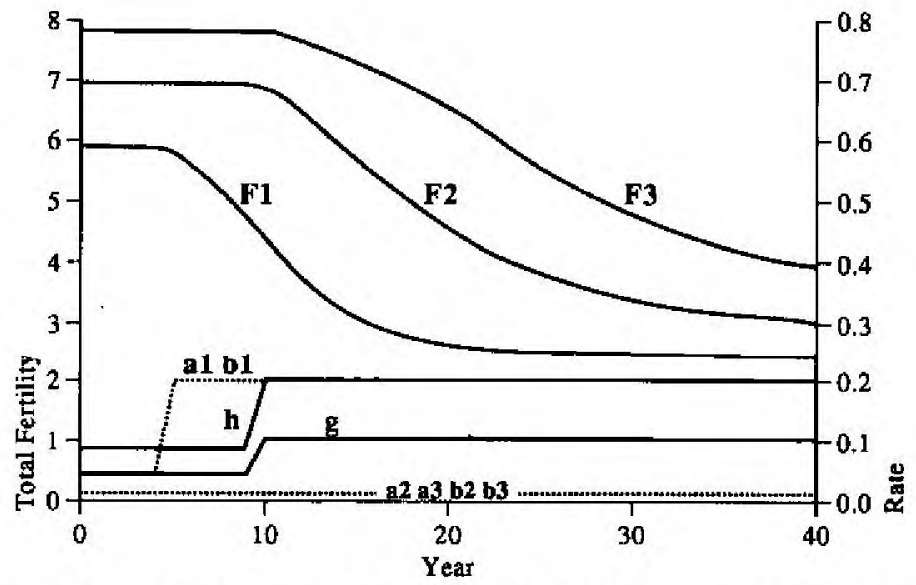

Figure 8. Simulating fertility transition trickle-down effects through three strata. Heterogeneous mix, interaction diffusion model. (a) Constant contact rates; $(b)$ increasing contact rates.

2 than in stratum 3 at all times. The differences in fertility between strata 2 and 3 can be attributed entirely to social interaction effects. Note that these effects are present throughout the simulation: in pre- and post-transition equilibria, as well as during the course of the decline. This result indicates that, where diffusion effects operate, crosssectional fertility differentials reflect, in part at least, social distance. Note, finally, that the size of the differential attributable to social interaction effects widens during the first stages of the transition and then attenuates during the later stages. (Consider how the distance between curves $F 2$ and $F 3$ varies over time.) In sum, the simulations suggest that contagion effects are observable throughout the period under observation (pretransition to post-transition equilibrium), but that the association between proximity to the source and the pace of fertility decline changes over the course of the transition.

A final point that emerges from Figure 8 is that the onset of fertility transition appears to be weakly associated with proximity to the source of contagion. Proximity to source is of much more consequence for the pace of transition and the post-transition level of fertility. 


\section{DISCUSSION}

The conventional models of fertility transition - what we have termed the demandsupply paradigm - implicitly assume isolated individuals. In these models, decisions about reproductive behaviour are determined through a weighting of the private benefits and costs of children and the objective and subjective costs of birth control. Routine behaviour dictated by cultural norms is also acknowledged in most conventional models. The fertility of a population is thus a simple aggregate of individual motivations and cost constraints. The assumption of isolated individuals would, of course, be rejected by most scholars. Nevertheless, systematic efforts to introduce more reasonable assumptions about social interaction processes are for the most part absent from the existing literature.

In this article, we have included social interaction patterns in formal models of fertility transition. The models thus capture a dynamic that is frequently termed 'diffusion of birth control'. There are several plausible behavioural mechanisms underlying this dynamic, all sharing the central feature that adoption of birth control by some individuals influences the likelihood of adoption by others. To be more concrete, the models assume that the contraceptive behaviour of couples of a certain socio-economic status, residing in a certain socio-economic context, exposed to a certain family planning programme service environment, will vary with the level of birth control among other couples with whom they have social contact. If this is, indeed, the case, then birthcontrol behaviour is 'contagious', and the fertility of a population is not the simple aggregate of isolated individual decisions, but the end-product of complex social interactions.

Theories of social imitation are not new in the social sciences. ${ }^{47}$ That the practice of birth control spreads from an elite to the masses has been noted since the earliest writings about changes in fertility. And during the past two decades, scholars investigating historical and contemporary fertility transitions have, with increasing frequency, invoked the notion of diffusion processes in their efforts to interpret puzzling features of the spatial-temporal patterns of fertility change. Indeed, in some of the recent literature structural change and diffusion dynamics are set against each other as contending explanations for the timing and pace of fertility change.

Our objective is to add rigour to the ongoing debate, in two respects: first, by

7 specifying how diffusion processes can be introduced into existing theoretical models of fertility; and, secondly, by illustrating numerically the nature and magnitude of plausible diffusion effects. The theoretical treatment of diffusion processes is based on a dynamic, multi-state model of birth control adoption that is similar to deterministic models used to describe the propagation of contagious diseases. The model describes a system consisting of three states: $(N)$ natural fertility, $(L)$ latent demand, and $(C)$ birth control. Demand factors (shaped by structural and cultural conditions) drive the flow between states $N$ and $L$; supply factors (shaped in part by programme interventions) drive the flow between states $L$ and $C$. Interaction diffusion is then introduced as a feedback from state $C$ : the rate of transition between states $N$ and $L$ and between states $L$ and $C$ depends in part on the relative number in state $C$, as a function of the 'adequate contact rate' (a concept borrowed from epidemiological models) between those in state $C$ and the other states. The model is intrinsically dynamic, an essential feature for investigation of the process of fertility transition.

The formal models that we specify contain a relatively small number of parameters and states, linked by a relatively simple set of differential equations. These provide the

" G. Tarde, The Laws of Imitation (New York: Holt, 1890). 
basis for numerical simulations of fertility transition that illustrate the nature and magnitude of social-interaction diffusion effects. The simulations demonstrate that social-interaction diffusion can, in theory, have a substantial impact on the course of fertility transition. In the simulations examined in this article, the impact is most evident in the magnitude of the overall fertility decline and, accordingly, in the pace of decline during the period of transition.

Varying the parameters of the model yields further insights:

(1) Diffusion effects are larger under more restrictive conditions (i.e. lower demand and higher costs of birth control) and, not surprisingly, in populations with higher contact rates.

(2) If the contact rate is high enough (i.e. populations that are socially integrated and in which individuals are extremely sensitive to the behaviour of others), fertility differentials fade away, and fertility change appears as primarily an imitation process.

(3) The existence of pre-transition clusters of moderate fertility is essentially incompatible with an unchanging potential for social-interaction diffusion. In populations where there is a long history of controlled fertility in certain strata, one must assume either that social-interaction diffusion makes no contribution to fertility decline, or that contact rates increase sharply during the transition. This result bolsters the arguments of some scholars that the expansion of communication and transport networks - the integration of relatively isolated small communities into larger regional, national, and international communities - is an important factor underlying fertility transition. ${ }^{48}$

(4) Simulations governed by models that allow for heterogeneous mixing in the population illustrate the effect of inter-strata distance (i.e. geographical and social distances) on fertility. Closeness to a contagion source tends to generate: $(a)$ differentials in fertility levels that are consistent in direction but vary in magnitude over time; $(b)$ differentials in the speed of transition that change substantially over time; and (c) hardly observable differentials in the onset of fertility transition.

(5) Perhaps most intriguing, the simulations illustrate the manner in which reduced birth-control costs can generate increased birth-control demand. Empirical evidence sometimes suggests that such effects exist, but they have been the source of some controversy because they do not fit conveniently into many existing theoretical models. Yet increased demand as a response to reduced costs follows directly from the feedback paths specified in our models. The numerical simulations succinctly demonstrate that $r$ such effects are to be expected if the valid model includes feedback paths of the form we specify.

Consideration of social-interaction diffusion dynamics raises many questions about the role of social structure in determining the course of fertility transition. Although a large body of literature stresses the effects of social structure on fertility, this literature is focused mainly on the role of social structure in determining the costs and benefits of childbearing (by determining the roles of women and children, for example). Caldwell's development of the concept of 'intergenerational wealth flows' is an excellent example of work within this tradition. ${ }^{49}$ Less attention has been given to the role of social structure in determining the power of social-interaction diffusion processes. Clearly diffusion effects of this type will materialize only if some degree of social interaction exists. Moreover, the strength of the diffusion effects is a direct function of the intensity

48 R. Freedman, 'Theories of fertility decline: a reappraisal', Social Forces, 58, 1 (1979), pp. 1-17. S. C. Watkins, 'From local to national communities: the transformation of demographic regimes in Western Europe, 1870-1960', Population and Development Review, 6. 2 (1990), pp. 241-272.

49 Caldwell, op, cit., fn. 2. 
of social interaction (more precisely, the 'adequate contact rate' - the interactions must have the potential of including exchanges about reproductive behaviour). We are largely in the dark about what types of social structures most facilitate high adequate contact rates. It is not clear, for example, that the potential is greater in highly integrated and compact societies, contrary to the prevailing assumption. ${ }^{50}$ Such societies may be more isolated from other societies (hence the opportunity for a diffusion process to get under way is reduced), and the force of existing norms may be more severe. As Crook ${ }^{51}$ has suggested, echoing an argument contained in Granovetter's influential paper, ${ }^{52}$ a society characterized by less tightly structured and integrated social relations - Granovetter's 'loose ties' society - may be more receptive to social-interaction diffusion processes. What is clear is that the potential for social-interaction diffusion is nil in societies where people do not talk to each other about reproductive matters, where women are secluded, and where communication among social strata and communities is minimal. Meaningful 'adequate contact rates' between and within strata are critical for interaction diffusion to operate.

This suggests the need for empirical research on the role of social structure in conditioning the strength of diffusion processes. Direct quantification of contact rates can be obtained through micro-level studies of social networks, surveys on frequency of interchanges on reproductive matters, and the observation of behavioural changes as product of such interchanges. These rates can then be related to the macro-level social structures that almost certainly have a determining influence. Information on the potential for social-interaction diffusion is also of considerable relevance to the design of population programmes. Clearly, efforts to plan, or to evaluate, interventions aimed at increasing birth control demand or reducing birth control costs should take into account the multiplier effects of diffusion. Moreover, the potential may even exist to use diffusion processes as a deliberate policy tool. In the early days of the family planning programme in Taiwan, for example, it appears that family planning fieldworkers instigated informal social-interaction diffusion that contributed significantly to the increase in knowledge and use of modern contraceptives. ${ }^{53}$

${ }^{50}$ E.g. Retherford, loc. cit., fn. 18.

${ }^{51} \mathrm{~N}$. Crook, 'On social norms and fertility decline', Journal of Development Studies, 14, 4 (1978), pp. $198-210$.

${ }_{52}$ M. S. Granovetter, 'The strength of weak ties', American Journal of Sociology, 78, 6 (1973), pp. $1360-1380$.

${ }^{53}$ R. Freedman and J. Y. Takeshita, Family Planning in Taiwan: An Experiment in Social Change (Princeton: Princeton University Press, 1969). 